\title{
Iron status and cardiovascular disease risk in black South African women: the PURE study
}

\author{
aAderibigbe OR, MSc, aPisa PT, PhD, a Mamabolo RL, PhD, a Kruger HS, PhD, a Vorster HH, DSc, bruger A, PhD \\ ${ }^{a}$ Centre of Excellence for Nutrition and ${ }^{\mathrm{b} A f r i c a}$ Unit for Transdisciplinary Health Research, North-West University, Potchefstroom \\ Correspondence to: Olaide R Aderibigbe, e-mail: connecttolaide@yahoo.com \\ Keywords: iron status, cardiovascular disease, African women, PURE study
}

\section{Abstract}

Objective: To examine the associations between measures of iron status and cardiovascular disease (CVD) risk factors in South African women.

Method: In a cross-sectional study, demographic information and health history were obtained during individual interviews using validated questionnaires in the North West Province, South Africa. Anthropometric indices, iron indices, blood pressure, blood glucose and lipid profiles were measured using standard procedures in 1262 apparently healthy black South African women aged 35 years or older. Iron status was assessed using serum concentrations of ferritin, transferrin receptor (TfR) and TfR:ferritin ratio.

Results: Associations between iron status parameters and CVD risk factors were generally weak $(r<0.3, p<0.01)$ and were not retained when adjustment was made for age, body mass index, smoking, alcohol consumption and C-reactive protein in the analysis. Waist circumference (WC) and waist:hip ratio (WHR) were higher in the fourth quartile of serum ferritin than in the third quartile, and also in the third quartile compared to the second quartile $(P<0.05)$. Based on WC and WHR respectively, $31 \%$ and $52 \%$ of the women had excess abdominal obesity. The mean ( $95 \%$ confidence interval) serum TfR concentration was high, at $9.09 \mu \mathrm{g} / \mathrm{ml}(8.77,9.44)$, indicating risk of iron deficiency. The mean (95\% confidence interval) concentrations of lipids [total cholesterol $4.78 \mathrm{mmol} / \mathrm{I}(4.64,4.93)$, high-density lipoprotein cholesterol $1.45 \mathrm{mmol} / \mathrm{I}(1.39,1.52)$, low-density lipoprotein cholesterol $1.65 \mathrm{mmol} / /(1.53,1.78)$, triglyceride $1.12 \mathrm{mmol} / \mathrm{l}(1.07,1.18)]$ were within reference ranges.

Conclusion: No significant association was found between iron status parameters and established CVD risk factors. However, excessive abdominal adiposity indicated by high WC and WHR contributes significantly to increased serum ferritin concentration in this population.

(P) Peer reviewed. (Submitted: 2011-03-29. Accepted: 2011-09-14.) ๑ SAJCN S Afr J Clin Nutr 2011;24(4):179-185

\section{Introduction}

The role that iron plays in the pathogenesis of cardiovascular disease (CVD) has increasingly been of interest in the field of public health. Excess iron has been proposed to catalyse the conversion of poorly reactive free radicals into highly reactive ones, a process known to predispose to or promote the incidence of CVD. ${ }^{1}$ At the moment, a number of iron supplementation or fortification programmes are ongoing in many developed and developing nations. Reports ${ }^{2}$ have shown that the percentage of iron-replete persons is on the increase in developed nations; reports also exist of iron overload in subgroups of people in the less developed nations. ${ }^{3}$ Moreover, there are speculations that iron overload may become a world epidemic. ${ }^{4}$

Most of the cross-sectional studies that examined the link between iron stores and increased CVD risk were conducted in men. ${ }^{5-11}$ In a study in women with poor iron status, a strong positive association was found between iron stores and CVD risk factors. ${ }^{2}$ This raises concern about the situation among African women. While undernutrition remains a problem, obesity and associated noncommunicable diseases, which were thought to be a problem of industrialised countries, are now increasingly becoming prevalent in the less industrialised countries, especially those undergoing transition. ${ }^{12-13}$ It has been proposed that iron overload may not be a problem in a healthy population, but that in persons with increased oxidative stress and obesity, iron overload may pose a greater risk. ${ }^{14-15}$

However, studies that have examined the role of iron status in CVD have been inconsistent, possibly because of the use of different measures of iron status. ${ }^{5-6,10-11}$ Iron status measured as dietary iron, serum iron, serum ferritin and transferrin saturation has limitations because these are influenced by short-term effects such as inflammation, iron intake, blood loss and diurnal variations. ${ }^{16-17}$ Soluble transferrin receptor (TfR) concentration has been suggested as an improved measure of iron status. ${ }^{18}$ Furthermore, because serum ferritin reflects the storage iron compartment and serum TfR reflects the functional iron compartment, the TfR:ferritin ratio has been suggested as a better estimate of body iron compared to either 
of the two measured independently..$^{19}$ Tuomainen et al ${ }^{8}$ reported that this ratio is virtually independent of inflammation. In view of this, the present study was aimed at using concentrations of serum ferritin, TfR and TfR:ferritin ratio to examine the association between iron status and CVD risk factors.

\section{Method}

\section{Study design, subject selection and organisational procedures}

This cross-sectional study was part of the North West province, South Africa (NWPSA) leg of the 12-year Prospective Urban and Rural Epidemiological (PURE) study. It investigated the effect of health transition on chronic diseases of lifestyle in urban and rural subjects. All the baseline data used for the present study were collected during 2005 in the North West. Migration stability was the main selection criterion within the chosen rural and urban communities.

The rural community (A) was identified $450 \mathrm{~km}$ west of Potchefstroom, on the highway to Botswana. A deep rural community (B), $35 \mathrm{~km}$ east from $A$ and only accessible via a gravel road, was also included. Community $\mathrm{C}$ was selected from the established part of the township next to Potchefstroom, and community $D$ was selected from the informal settlements surrounding community $\mathrm{C}$. A household census regarding number of people, their ages and health profile was randomly done in 6000 houses (1500 in each community).

The head of each household gave signed consent to complete the questionnaire. If a person refused or was not at home, the next house was taken and a non-complier questionnaire was completed. From the data obtained, a paper selection of possible subjects with no reported chronic diseases of lifestyle, tuberculosis or diagnosed human immunodeficiency virus (HIV) infection was made. A total of 2010 (35 years and older) apparently healthy blackAfrican volunteers were recruited. The sample for the study described in this article consisted of only the 1262 female participants. Data collection was divided into stations. Station 1 involved the administration of questionnaires, station 2 involved anthropometric measurements and blood pressure (BP) measurements, and station 3 involved blood collection. Participants were fasted (10-12 hours) for the baseline blood sampling and other measurements. Trained field workers assisted in providing information to the participants in their language of choice. Participants received feedback regarding their BP, fasting blood glucose concentrations and HIV status and were referred to counselling services, clinics or hospitals where necessary. Travel expenses of participants were covered.

Ethical approval was obtained from the Ethics Committee of the North-West University, Potchefstroom, South Africa (ethics number: 04M10), and signed informed consent forms were obtained from all participants. Permission to conduct the study was given by the North West Department of Health, tribal chiefs, community leaders, employers and mayors.

\section{Questionnaires}

Structured, validated demographic, socio-economic and lifestyle questionnaires were administered by trained field workers during home visits in the language preferred by the participants. Adapted questionnaires were used by all countries participating in the PURE study.

\section{Anthropometric measurements}

Anthropometric measurements were carried out by biokineticists. Height was measured to the nearest $0.5 \mathrm{~cm}$ with a stadiometer (Invicta, IP 1465, United Kingdom), and weight was determined on portable electronic scales to the nearest $0.01 \mathrm{~kg}$ (Precision Health Scale, A \& D Company, Japan). All the measurements were conducted according to the guidelines adopted at the National Institute of Healthsponsored Arlie Conference..$^{20}$ Body circumferences of participants were measured in light underwear with calibrated instruments (Holtain ${ }^{\circledR}$ unstretchable metal tape, John Bull ${ }^{\circledR}$ calipers). Body mass index (BMI) was calculated by dividing weight in kilograms by height in metres squared. Waist:hip ratio (WHR) was calculated by dividing waist circumference (WC) by hip circumference in centimetres.

\section{HIV status}

Everyone who signed an informed consent form was tested for HIV infection, but was given the choice of knowing her status. Whole blood was used for the determination of HIV status with the First Response $^{\circledR}$ (PMC Medical, India) rapid HIV card test. If tested positive, the test was repeated with the Pareeshak ${ }^{\circledR}$ (BHAT Biotech India) card test. Pre-test counselling was done in groups of 10 persons before the blood sample was taken, and individual posttest counselling was done according to the protocol of the National Department of Health of South Africa. ${ }^{21}$

\section{Blood, serum and plasma samples}

A disposable needle was used to draw blood from the antecubital vein in the right arms of participants. The blood collection tubes were appropriately filled in order to ensure optimal blood-to-anticoagulant ratios. Each tube was inverted gently five times for thorough mixing. Samples were placed in ice boxes after proper labelling. A new sterile-transfer pipette was used for aliquotting blood samples for analysis. Serum was prepared by allowing blood to clot at room temperature for 30 minutes; it was then centrifuged at $2000 \mathrm{~g}$ for 15 minutes at $10{ }^{\circ} \mathrm{C}$. Blood was centrifuged within two hours of collection. All blood and serum samples were stored at $-70{ }^{\circ} \mathrm{C}$ in the laboratory. Plasma samples were collected in ethylenediaminetetraacetic acid (EDTA) tubes, centrifuged at $2000 \mathrm{~g}$ for 15 minutes at 4 ${ }^{\circ} \mathrm{C}$ and transferred to cryo tubes for storage at $-70{ }^{\circ} \mathrm{C}$.

\section{Biochemical and physiological analyses}

Systolic BP and diastolic BP were measured with the validated OMRON HEM-757 device (Omron HEM-757). After a 10-minute rest period, BP measurements were performed twice (five minutes apart) on the right arm (brachial artery), while the participants were seated upright and relaxed with the right arm supported at heart level. Plasma glucose was measured using Vitros ${ }^{\circledR}$ DT6011 Chemistry Analyser, an Ortho-Clinical Diagnostics tool (Rochester, New York, United States of America). Quantitative determination of total cholesterol (TC), highdensity lipoprotein cholesterol (HDL-C), triglyceride (TG) and highsensitivity C-reactive protein (CRP) concentration in serum was done by Sequential Multiple Analyser Computer (SMAC) using the Konelab $20 \mathrm{i}^{\circledR}$ auto analyser (Thermo Fisher Scientific Oy, Vantaa, Finland). Low-density lipoprotein cholesterol (LDL-C) was calculated using the Friedewald formula. ${ }^{22}$ Serum ferritin and TfR concentrations were determined quantitatively using an enzyme immunoassay procedure 
(Ramco Laboratories, Inc, Stafford Texas). The coefficient of variance (CV) for all assays was less than $10 \%$.

\section{Statistical analysis}

Data were analysed using version 17 of the Statistical Package for Social Sciences ${ }^{\circledR}$ (SPSS Inc Chicago III, September 2008). Data were not normally distributed and were log transformed to improve normality. Data were presented as mean [95\% confidence interval $(\mathrm{Cl})$. Pearson correlation was used to assess the associations between iron status parameters and CVD risk factors. In this study, the following CVD risk factors were examined: BMI, WC, WHR, TC, LDL-C, HDL-C, TG, systolic and diastolic BP, plasma glucose and CRP. Age, BMl, smoking, alcohol consumption and serum CRP were treated as valid confounders. Association was further assessed by a partial correlation, adjusting for these confounders. For further assessment of the relationship between iron status and CVD risk factors, participants were grouped and analysed in quartiles of ferritin, TfR and TfR:ferritin ratio. Multivariate analysis was used to assess significant differences between different quartiles before and after adjusting for valid confounders. A $\mathrm{P}<0.05$ was considered statistically significant. HIV status did not confound or modify the associations between iron indices and CVD risk factors; hence, it was not treated as a valid confounder.

Table I: Selected characteristics of women participants in the PURE study $(\mathrm{n}=1262)$

\begin{tabular}{|l|c|}
\hline Variable & $\begin{array}{c}\text { Mean }(95 \% \text { confidence } \\
\text { interval) }\end{array}$ \\
\hline Age (years) & $49.16(48.57,49.76)$ \\
\hline Smoking status (cigarettes/day) & $2.61(2.38,2.85)$ \\
\hline Alcohol consumption (g/day) & $7.74(6.59,8.89)$ \\
\hline Body mass index (kg/m²) & $23.87(23.22,24.54)$ \\
\hline Waist circumference (cm) & $76.38(75.16,77.80)$ \\
\hline Waist:hip ratio & $0.80(0.79,1.81)$ \\
\hline TfR ( $\mu$ g/ml) & $9.09(8.77,9.44)$ \\
\hline Ferritin ( $\mu$ g/l) & $94.84(81.84,110-15)$ \\
\hline TfR:ferritin ratio & $0.25(0.19,0.30)$ \\
\hline Total cholesterol (mmol/l) & $4.78(4.64,4.93)$ \\
\hline High density lipoprotein cholesterol (mmol/l) & $1.45(1.39,1.52)$ \\
\hline Triglyceride (mmol/l) & $1.12(1.07,1.18)$ \\
\hline Low density lipoprotein cholesterol (mmol/l) & $1.65(1.53,1.78)$ \\
\hline Diastolic blood pressure blood pressure & $85.90(84.52,87.49)$ \\
\hline mmHg) & \\
\hline Systolic blood pressure blood pressure (mmHg) & $127.93(125.60,130.31)$ \\
\hline Glucose (mmol/l) & $5.34(5.21,5.47)$ \\
\hline C-reactive protein (mg/l) & $2.81(1.84,3.98)$ \\
\hline
\end{tabular}

\section{Results}

Table I outlines selected characteristics of women participants in the PURE study. The mean values of variables were within recommended reference ranges, ${ }^{23-27}$ except for TfR and WHR, which were higher, indicating risk for iron deficiency and abdominal obesity respectively in this population.
Table II illustrates the proportion (\%) of women according to standard cutoff points for variables and the proportion of self-reported smokers and alcohol consumers. Half $(50 \%)$ of the participants were selfreported smokers while $33 \%$ were alcohol consumers. In addition, $53 \%$ were either overweight or obese $\left(>25 \mathrm{~kg} / \mathrm{m}^{2}\right)$, while $31 \%$ had excess WC $(\geq 88 \mathrm{~cm})$ and $52 \%$ had excess WHR $(\geq 0.80)$. As for iron indices, $56 \%$ had a high TfR concentration $(>8.5 \mu \mathrm{g} / \mathrm{ml}$ ) while $33 \%$ had excess iron stores as defined by ferritin concentration (> $150 \mu \mathrm{g} / \mathrm{l})$. Those at risk as defined by increased biological health markers were $48 \%$ for TC, $27 \%$ for LDL-C, $23 \%$ for TG, $27 \%$ for glucose, $25 \%$ for CRP and $43 \%$ for HDL-C. Diastolic BP and systolic BP were increased in $57 \%$ and $49 \%$ of the women respectively.

Table II: Proportion of women participants in the PURE study according to standard cutoff points, smoking and alcohol consumption status

\begin{tabular}{|c|c|c|}
\hline Variable & $\begin{array}{l}\text { Recommended } \\
\text { reference value }\end{array}$ & n (\%) \\
\hline $\begin{array}{l}\text { Smokers } \\
\text { Non-smokers }\end{array}$ & & $\begin{array}{l}437(50 \%) \\
430(50 \%)\end{array}$ \\
\hline $\begin{array}{l}\text { Alcohol consumers } \\
\text { Alcohol non-consumers }\end{array}$ & & $\begin{array}{l}391(33 \%) \\
787(67 \%)\end{array}$ \\
\hline Body mass index ${ }^{23}$ & $\begin{array}{c}<18 \mathrm{~kg} / \mathrm{m}^{2} \\
18-25 \mathrm{~kg} / \mathrm{m}^{2} \\
\geq 25 \mathrm{~kg} / \mathrm{m}^{2}\end{array}$ & $\begin{array}{l}137(12 \%) \\
410(35 \%) \\
641(53 \%)\end{array}$ \\
\hline Waist circumference ${ }^{23}$ & $\begin{array}{l}<88 \mathrm{~cm} \\
\geq 88 \mathrm{~cm}\end{array}$ & $\begin{array}{l}808(69 \%) \\
368(31 \%)\end{array}$ \\
\hline Waist:hip ratio ${ }^{23}$ & $\begin{array}{l}<0.80 \\
\geq 0.80\end{array}$ & $\begin{array}{l}534(48 \%) \\
578(52 \%)\end{array}$ \\
\hline $\mathrm{TfR}^{24}$ & $\begin{array}{l}<8.5 \mu \mathrm{g} / \mathrm{ml} \\
\geq 8.5 \mu \mathrm{g} / \mathrm{ml}\end{array}$ & $\begin{array}{l}462(44 \%) \\
597(56 \%)\end{array}$ \\
\hline Ferritin ${ }^{25}$ & $\begin{array}{c}<12 \mu \mathrm{g} / \mathrm{l} \\
12-150 \mu \mathrm{g} / \mathrm{l} \\
>150 \mu \mathrm{g} / \mathrm{l})\end{array}$ & $\begin{array}{c}94(9 \%) \\
610(58 \%) \\
355(33 \%)\end{array}$ \\
\hline Total cholesterol ${ }^{26}$ & $\begin{array}{l}\leq 5 \mathrm{mmol} / \mathrm{l} \\
>5 \mathrm{mmol} / \mathrm{l}\end{array}$ & $\begin{array}{l}612(52 \%) \\
564(48 \%)\end{array}$ \\
\hline High-density lipoprotein cholesterol ${ }^{26}$ & $\begin{array}{l}\geq 1.3 \mathrm{mmol} / \mathrm{l} \\
<1.3 \mathrm{mmol} / /\end{array}$ & $\begin{array}{l}666(57 \%) \\
506(43 \%)\end{array}$ \\
\hline Triglyceride $^{26}$ & $\begin{array}{l}\leq 1.7 \mathrm{mmol} / \mathrm{l} \\
>1.7 \mathrm{mmol} / /\end{array}$ & $\begin{array}{l}904(77 \%) \\
265(23 \%)\end{array}$ \\
\hline Low-density lipoprotein cholesterol ${ }^{26}$ & $\begin{array}{l}\leq 3.0 \mathrm{mmol} / \mathrm{l} \\
>3.0 \mathrm{mmol} / \mathrm{l}\end{array}$ & $\begin{array}{l}754(73 \%) \\
281(27 \%)\end{array}$ \\
\hline Diastolic blood pressure ${ }^{26}$ & $\begin{array}{l}\leq 85 \mathrm{mmHG} \\
>85 \mathrm{mmHG}\end{array}$ & $\begin{array}{l}501(43 \%) \\
670(57 \%)\end{array}$ \\
\hline Systolic blood pressure ${ }^{26}$ & $\begin{array}{l}\leq 130 \mathrm{mmHg} \\
>130 \mathrm{mmHg}\end{array}$ & $\begin{array}{l}593(51 \%) \\
578(49 \%)\end{array}$ \\
\hline Glucose $^{26}$ & $\begin{array}{l}\leq 6.1 \mathrm{mmol} / \mathrm{l} \\
>6.1 \mathrm{mmol} / \mathrm{l}\end{array}$ & $\begin{array}{l}851(73 \%) \\
316(27 \%)\end{array}$ \\
\hline C-reactive protein ${ }^{27}$ & $\begin{array}{l}\leq 10 \mathrm{mg} / \mathrm{l} \\
>10 \mathrm{mg} / \mathrm{l}\end{array}$ & $\begin{array}{l}874(75 \%) \\
296(25 \%)\end{array}$ \\
\hline
\end{tabular}

The correlations between iron indices and CVD risk factors are shown in Table III. Ferritin correlated positively with TC, HDL-C, LDL-C, TG, diastolic BP, systolic BP, CRP and alcohol consumption before adjusting for valid confounders (age, BMI, smoking, alcohol consumption and CRP), but the correlations were not retained after adjustment. There was a negative correlation between TfR and 
LDL-C, and TG and alcohol consumption. A positive correlation was found between TfR and WHR. All of these disappeared after adjusting for valid confounders. TfR:ferritin ratio correlated negatively with TC, LDL-C, TG, diastolic BP, systolic BP, CRP and alcohol consumption before adjusting for valid confounders, but was not retained after adjustment. The correlations observed between iron indices and CVD risk factors were generally weak $(r<0.3)$.

CVD risk factors are compared according to ferritin quartiles in Table IV. BMI and WC were higher in quartile three than in quartile one before adjusting for valid confounders. After adjusting for the valid confounders, WC in the third quartile remained significantly higher than in the second and fourth quartiles. WHR increased across ferritin quartiles before adjusting for valid confounders. After adjustment for valid confounders, the increase from quartile two to quartile four remained significant. TC, TG, LDL-C, diastolic BP and systolic BP were significantly higher in the fourth quartile than in the first two quartiles before adjusting for valid confounders, but were not retained after adjustment. A significantly higher HDL-C value was observed in quartile four than in quartile one before adjusting for confounders. No significant difference was observed for blood glucose across ferritin quartiles. CRP was higher in quartiles two, three and four than in quartile one.

Table V shows the comparison of CVD risk factors according to TfR quartiles. BMI, WC, HDL-C, systolic BP, glucose and CRP did not vary significantly among the quartiles. WHR was significantly higher in quartiles three and four than in quartile two before adjusting for age, BMI, smoking, alcohol consumption and CRP. In addition, WHR was higher in quartile two than in quartile one before adjusting for
Table III: Correlations between iron indices and cardiovascular disease risk factors of participants

\begin{tabular}{|c|c|c|c|c|c|c|}
\hline \multirow[b]{2}{*}{ Variables } & \multicolumn{2}{|c|}{ Ferritin ( $\mu \mathrm{g} / \mathrm{L})$} & \multicolumn{2}{|c|}{ TfR $(\mu \mathrm{g} / \mathrm{ml})$} & \multicolumn{2}{|c|}{ TfR:ferritin ratic } \\
\hline & ${ }^{a} \mathbf{r}$ & ${ }^{{ }^{b}} r_{p}$ & $\mathbf{r}$ & $\mathbf{r}_{\mathrm{p}}$ & $\mathbf{r}$ & $\mathbf{r}_{\mathrm{p}}$ \\
\hline Ferritin $(\mu \mathrm{g} / \mathrm{l})$ & -- & -- & $-.351^{c}$ & -.079 & $-.776^{c}$ & $-.705^{c}$ \\
\hline $\operatorname{TfR}(\mu \mathrm{g} / \mathrm{ml})$ & $351^{c}$ & -.079 & -- & -- & $.511^{c}$ & $.410^{\circ}$ \\
\hline TfR:ferritin ratio & $.776^{c}$ & $-.705^{c}$ & $.511^{\mathrm{c}}$ & $.410^{\mathrm{c}}$ & -- & -- \\
\hline Body mass index $\left(\mathrm{kg} / \mathrm{m}^{2}\right)$ & -.059 & -- & .009 & --- & -.025 & -- \\
\hline Waist circumference $(\mathrm{cm})$ & .029 & -.022 & .003 & -.019 & -.058 & .056 \\
\hline Waist:hip ratio & .049 & .071 & $.124^{c}$ & .014 & .017 & -.067 \\
\hline Total cholesterol (mmol/l) & $.065^{d}$ & -.037 & -.031 & -.091 & $-.072^{d}$ & -.017 \\
\hline $\begin{array}{l}\text { High density } \\
\text { cholesterol ( } r\end{array}$ & $065^{\mathrm{d}}$ & -.046 & -.037 & 02 & 5 & .028 \\
\hline Triglyceride (mmol/l) & $.187^{\circ}$ & .034 & $-.075^{d}$ & -.079 & $-.120^{c}$ & -.042 \\
\hline $\begin{array}{l}\text { Low density lipoprotein } \\
\text { cholesterol (mmol/l) }\end{array}$ & $.140^{\circ}$ & .012 & $-.063^{d}$ & -.157 & $-.109^{c}$ & -.053 \\
\hline $\begin{array}{l}\text { Diastolic blood pressure } \\
(\mathrm{mmHg})\end{array}$ & $.182^{c}$ & .068 & -.035 & -.055 & $-.139^{c}$ & -.084 \\
\hline $\begin{array}{l}\text { Systolic blood pressure } \\
(\mathrm{mmHg})\end{array}$ & $.169^{c}$ & .011 & -.048 & -.097 & $-.116^{c}$ & -.086 \\
\hline Glucose (mmol/l) & .019 & -.007 & .001 & .048 & .018 & -.013 \\
\hline C-reactive protein (mg/l) & $.158^{\mathrm{c}}$ & -- & .029 & -- & $-.133^{c}$ & -- \\
\hline Smoking (cigarette/day) & .008 & -- & -.078 & -- & -.008 & -- \\
\hline $\begin{array}{l}\text { Alcohol consumption } \\
\text { (g/day) }\end{array}$ & $.207^{\mathrm{c}}$ & -- & $-.109^{d}$ & -- & $-.147^{c}$ & - \\
\hline
\end{tabular}

$a=$ Pearson correlation coefficient after adjusting for age, body mass index, smoking, alcohol consumption and $\mathrm{C}$-reactive protein, $\mathrm{b}=$ partial correlation coefficient after adjusting for age, body mass index, smoking, alcohol consumption and C-reactive protei, $c=$ Correlation is significant at the 0.01 level (two-tailed), $d=$ Correlation is significant at the 0.05 level (two-tailed)

Table IV: Mean (95\% confidence interval) of CVD risk factors according to ferritin quartiles

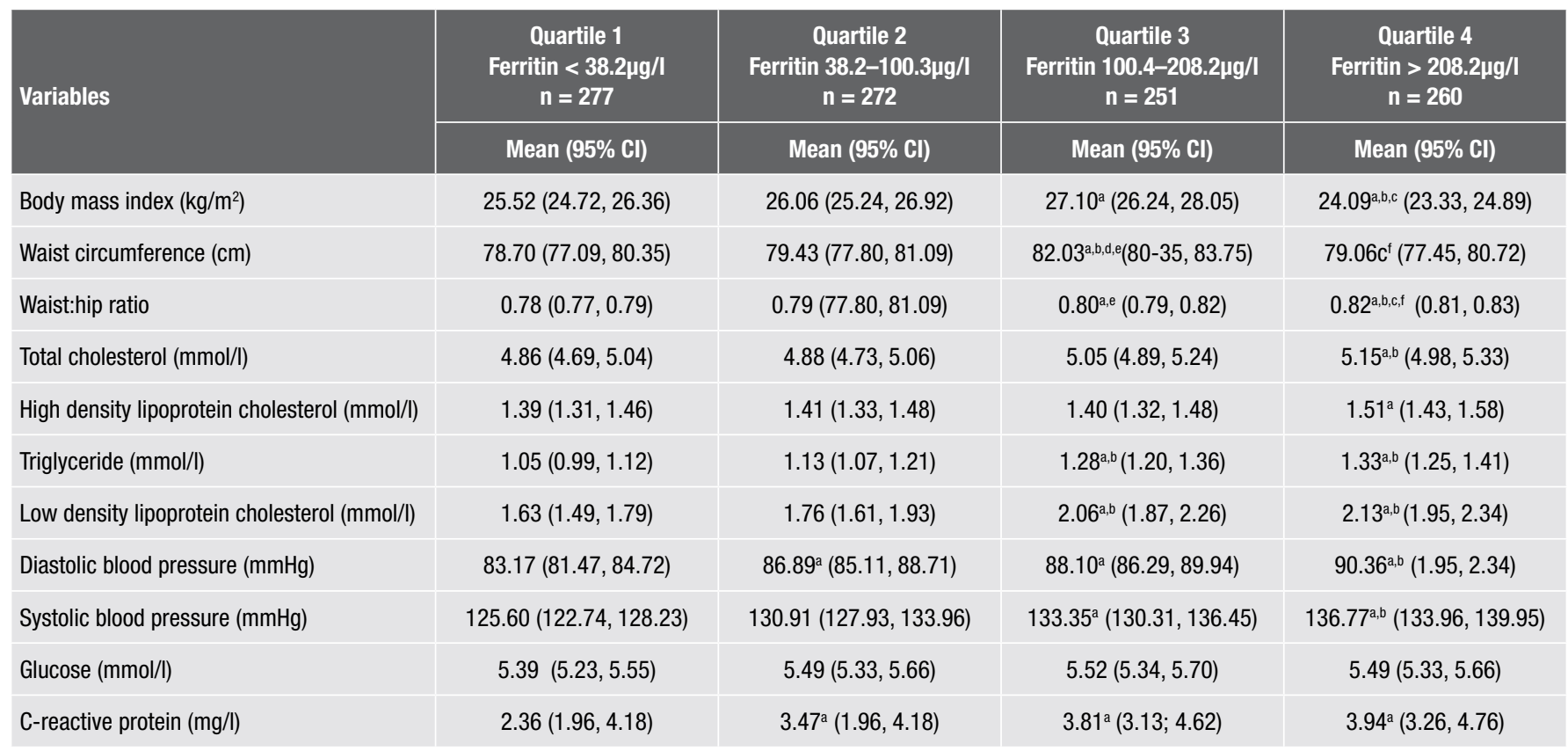

aP $<0.05$ significantly different from quartile 1 (unadjusted)

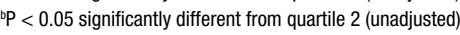

iP $<0.05$ significantly different from quartile 3 (unadjusted)

aP $<0.05$ sinificantly different from quartile 1 (adjusted for age, body mass index, smoking, alcohol consumption and C-reactive protein)

eP $<0.05$ significantly different from quartile 2 (adjusted for age, body mass index, smoking, alcohol consumption and C-reactive protein)

I $P<0.05$ significantly different from quartile 3 (adjusted for age, body mass index, smoking, alcohol consumption and C-reactive protein) 
Table V: Mean (95\% confidence interval) of CVD risk factors according to TfR quartiles

\begin{tabular}{|c|c|c|c|c|}
\hline Variables & $\begin{array}{c}\text { Quartile } 1 \\
\text { TfR }<7.8 \mu \mathrm{g} / \mathrm{I} \\
n=374\end{array}$ & $\begin{array}{c}\text { Quartile } 2 \\
\text { TfR 7.8-9.7 } \mathrm{pg} / \mathrm{l} \\
n=262\end{array}$ & $\begin{array}{c}\text { Quartile } 3 \\
\text { TfR } 9.8-11.9 \mu g / l \\
n=216\end{array}$ & $\begin{array}{c}\text { Quartile } 4 \\
\text { TfR }>11.9 \mu \mathrm{g} / \mathrm{l} \\
n=208\end{array}$ \\
\hline & Mean $(95 \%$ CI) & Mean $(95 \% \mathrm{CI})$ & Mean $(95 \%$ Cl) & Mean $(95 \%$ CI) \\
\hline Body mass index $\left(\mathrm{kg} / \mathrm{m}^{2}\right)$ & $25.29(24.60,26.06)$ & $25.82(24.94,26.66)$ & $25.82(24.88,26.79)$ & $25.94(25.00,26.91)$ \\
\hline Waist circumference (cm) & $79.43(77.98,80.90)$ & $79.43(77.80,81.09)$ & $79.98(78.16,81.84)$ & $80.72(78.88,82.60)$ \\
\hline Waist:hip ratio & $0.80(0.79,0.81)$ & $0.78^{\mathrm{a}}(0.77,0.79)$ & $0.81^{b}(0.79,0.81)$ & $0.80^{b}(0.79,0.81)$ \\
\hline Total cholesterol (mmol/l) & $5.04(4.89,5.19)$ & $4.88^{\mathrm{c}}(4.72,5.05)$ & $5.06^{c}(4.88,5.27)$ & $4.94(4.75,5.12)$ \\
\hline High density lipoprotein cholesterol ( $\mathrm{mmol} / \mathrm{l})$ & $1.42(1.36,1.48)$ & $1.43(1.34,1.50)$ & $1.46(1.38,1.55)$ & $1.40(1.32,1.48)$ \\
\hline Triglyceride (mmol/l) & $1.27(1.21,1.34)$ & $1.13^{\mathrm{a}}(1.06,1.20)$ & $1.14^{\mathrm{a}}(1.07,1.22)$ & $1.19(1.11,1.27)$ \\
\hline Low density lipoprotein cholesterol (mmol/l) & $2.05(1.89,2.22)$ & $1.71^{\mathrm{a}}(1.56,1.88)$ & $1.84(1.66,2.04)$ & $1.85(1.67,2.06)$ \\
\hline Diastolic blood pressure (mmHg) & $86.49(85.11,88.10)$ & $87.49(85.70,89.33)$ & $86.89(84.91,88.92)$ & $87.09^{\mathrm{a}, \mathrm{d}}(85.11,89.12)$ \\
\hline Glucose (mmol/l) & $5.40(5.26,5.54)$ & $5.49(5.33,5.66)$ & $5.38(5.19,5.57)$ & $5.66(5.47,5.86)$ \\
\hline C-reactive protein (mg/l) & $3.13(2.66,3.68)$ & $3.29(2.72,3.98)$ & $3.15(5.19,5.57)$ & $3.89(5.47,5.86)$ \\
\hline
\end{tabular}

ap $<0.05$ significantly different from quartile 1 (unadjusted)

${ }^{\mathrm{bP}}<0.05$ significantly different from quartile 2 (unadjusted)

"P $<0.05$ significantly different from quartile 1 (adjusted for age, body mass index, smoking, alcohol consumption and C-reactive protein)

dP $<0.05$ significantly different from quartile 2 (adjusted for age, body mass index, smoking, alcohol consumption and C-reactive protein)

Table VI: Mean (95\% confidence interval) of CVD risk factors according to TfR:ferritin ratio quartiles

\begin{tabular}{|c|c|c|c|c|}
\hline Variables & $\begin{array}{c}\text { Quartile } 1 \\
\text { TfR:ferritin }<0.04 \\
n=256\end{array}$ & $\begin{array}{c}\text { Quartile 2 } \\
\text { TfR:ferritin 0.04-0.09 } \\
n=263\end{array}$ & $\begin{array}{c}\text { Quartile 3 } \\
\text { TfR:ferritin } 0.10-0.25 \\
n=281\end{array}$ & $\begin{array}{c}\text { Quartile } 4 \\
\text { TfR:ferritin } 0.10-0.25 \\
n=281\end{array}$ \\
\hline & Mean (95\% CI) & Mean (95\% Cl) & Mean $(95 \% \mathrm{Cl})$ & Mean (95\% CI) \\
\hline Body mass index $\left(\mathrm{kg} / \mathrm{m}^{2}\right)$ & $24.09(23.33,24.88)$ & $26.97^{\mathrm{a}}(26.06,27.86)$ & $26.18^{\mathrm{a}}(25.35,27.03)$ & $25.52^{\mathrm{a}, \mathrm{c}}(24.66,26.36)$ \\
\hline Waist circumference $(\mathrm{cm})$ & $79.06(77.44,80.72)$ & $81.65^{\mathrm{a}, \mathrm{b}}(79.98,83.36)$ & $79.61(77.98,81.28)$ & $78.88^{\mathrm{c}}(77.26,80.72)$ \\
\hline Waist:hip ratio & $0.82(0.81,0.83)$ & $0.80^{\mathrm{a}}(0.79,0.81)$ & $0.79^{\mathrm{a}}(0.78,0.80)$ & $0.78^{\mathrm{a}}(0.78,0.80)$ \\
\hline Total cholesterol (mmol/l) & $5.15(4.97,5.33)$ & $4.97(4.80,5.15)$ & $4.94(4.78,5.11)$ & $4.88^{\mathrm{a}}(0.77,0.80)$ \\
\hline High density lipoprotein cholesterol (mmol/l) & $1.51(1.44,1.60)$ & $1.39^{\mathrm{a}}(1.31,1.46)$ & $1.39^{\mathrm{a}}(1.32,1.47)$ & $1.40^{\mathrm{a}}(1.33,1.48)$ \\
\hline Triglyceride (mmol/l) & $1.37(1.32,1.46)$ & $1.22^{\mathrm{a}}(1.31,1.46)$ & $1.14^{\mathrm{a}}(1.07,1.21)$ & $1.06^{\mathrm{a}, \mathrm{c}}(1.00,1.13)$ \\
\hline Low density lipoprotein cholesterol (mmol/l) & $2.18(1.99,2.40)$ & $1.93(1.75,2.12)$ & $1.79^{\mathrm{a}}(1.64,1.97)$ & $1.65^{\mathrm{a}, \mathrm{c}}(1.50,1.81)$ \\
\hline Diastolic blood pressure (mmHg) & $90.15(88.30,92.04)$ & $88.10(86.29,89.94)$ & $86.49^{\mathrm{a}}(84.91,88.30)$ & $83.36^{\mathrm{a}, \mathrm{c}, \mathrm{d}}(81.65,85.11)$ \\
\hline Systolic blood pressure (mmHg) & $136.14(133.35,139.31)$ & $133.65(86.29,89.94)$ & $130.91^{\mathrm{a}}(128.23,133.96)$ & $125.60^{\mathrm{a}, \mathrm{c}, \mathrm{d}}(122.74,128.52)$ \\
\hline Glucose $(\mathrm{mmol} / \mathrm{l})$ & $5.53(5.35,5.70)$ & $5.38(5.22,5.55)$ & $5.52(5.35,5.68)$ & $5.45(5.28,5.62)$ \\
\hline C-reactive protein (mg/l) & $3.87(3.21,4.68)$ & $3.79(3.13,4.60)$ & $3.40(2.83,4.09)$ & $2.42^{\mathrm{a}, \mathrm{c,d}}(2.00,2.92)$ \\
\hline
\end{tabular}

aP $<0.05$ significantly different from quartile 1 (unadjusted)

bP $<0.05$ significantly different from quartile 1 (adjusted for age, body mass index ,smoking, alcohol consumption and C-reactive protein)

CP $<0.05$ significantly different from quartile 2 (unadjusted)

up $<0.05$ significantly different from quartile 3 (unadjusted)

valid confounders. A significantly higher diastolic BP was observed in quartile four than in quartiles one and three after adjusting for valid confounders. LDL-C was only lower in quartile two than in quartile one before adjustment while TC became significantly higher in quartiles two and three than in quartile one after adjusting for valid confounders.

Table VI compares CVD risk factors among TfR:ferritin ratio quartiles. A significantly higher BMI was observed in quartiles two, three and four than in quartile one. There were consistently lower values of
WHR, TC, HDL-C, TG, LDL-C diastolic BP, systolic BP and CRP in the fourth quartile than in the first quartile before adjusting for valid confounders. Only WC in quartile two was significantly higher than in quartile one after adjusting for valid confounders

\section{Discussion}

This study examined the associations between iron status and CVD risk factors in black South African women. Ferritin was positively associated with TC $(p<0.05)$, HDL-C $(p<0.05)$, TG $(p<0.01)$, 
LDL-C $(p<0.01)$, diastolic and systolic BP $(p<0.01)$, CRP $(p<0.01)$ and alcohol consumption $(p<0.01)$. TfR:ferritin ratio was negatively associated with the same variables as ferritin except for HDL-C. TfR was negatively associated with TC, LDL-C and alcohol consumption but positively associated with WHR. None of these associations were retained after adjusting for age, BMI, smoking, alcohol consumption and CRP. WC and WHR were significantly higher in the fourth quartile of ferritin than in the third quartile after adjustment. In addition, WC and WHR in the third quartile of ferritin were higher than in the second quartile after adjusting for valid confounders.

The hypothesis that excess iron is causally linked to CVD dates as far back as the 1980s, when Sullivan suggested that increased incidence of CVD in men and postmenopausal women was due to increased body iron..$^{28}$ Free iron can cause the oxidation of LDL-C, thereby making it highly cardiotoxic. ${ }^{29}$ Oxidised LDL-C has the ability to cause direct injury to endothelial cells, leading to generation of foam cells. ${ }^{29}$ However, the results of this study do not support this hypothesis (of an association between excess iron and CVD risk), because the observed associations could be attributed to the effects of other factors such as age, BMI, smoking, alcohol consumption and infection on iron stores. Though very weak, WC, WHR, TC, TG, and diastolic BP were positively associated with ferritin when age, BMI, smoking and CRP only were adjusted for in the analysis. The inclusion of alcohol consumption on the list of valid confounders removed this association. This indicates that alcohol consumption is a strong predictor of iron status in this population, as confirmed by the study conducted by Gopane ${ }^{30}$ in the same population. Furthermore, this population seems to be at risk of iron deficiency, as indicated by a mean serum TfR concentration of $9.09 \mu \mathrm{g} / \mathrm{ml}(95 \%$ $\mathrm{Cl} 8.77,9.44)$, which could explain the lack of significant association between measures of iron status and the CVD risk factors in the present study. Additionally, $56 \%$ of the participants had a mean serum TfR concentration greater than $8.50 \mu \mathrm{g} / \mathrm{ml}$, indicating iron deficiency, as compared to $33 \%$ with increased serum ferritin concentration (ferritin $>150 \mu \mathrm{g} / \mathrm{l}$ ), indicating iron overload. It is also possible that the associations were not significant because most of the women were not hypercholesterolaemic ( $52 \%$ had TC less than or equal to $5 \mathrm{mmol} / \mathrm{l}$ ).

The findings of the present study do not agree with those of some studies that have examined the relationship between iron status and CVD risk factors. ${ }^{6,31}$ These studies demonstrated a positive association between serum ferritin and TC concentration. Postmenopausal women were used as participants in these studies, which makes their results less comparable to those of the present study, which was composed of adult women both of pre- $(76 \%)$ and postmenopausal (24\%) age (over 55 years). Menopause itself has been reported to result in increased iron storage and lipid production, owing to the absence of the protective effect of endogenous oestrogen. ${ }^{32}$ Additionally, a positive association was found between serum ferritin and TG, but not TC, HDL-C and BP, in a healthy population of white Danish women aged 40-60 years. ${ }^{33}$ In the present study, TG was positively associated with ferritin only before adjusting for valid confounders. It has been proposed that increased serum TG may cause an increase in the release of small LDL particles through lipid transfer reaction. ${ }^{34}$ Small LDL particles are easily oxidised, the initial step in CVD development. ${ }^{35}$ Increases in small and dense LDL particles have been linked to increased CVD risk. ${ }^{35}$

The findings of the present study are comparable to the observations made by Binkoski et al, who reported that increased body iron does not increase the susceptibility of LDL to oxidative stress in women with low iron status. ${ }^{36} \mathrm{~A}$ meta-analysis also confirmed that increased iron stores do not play a significant role in the development of CVD. ${ }^{37}$ Additionally, the Atherosclerotic Risk in Communities (ARIC) study ${ }^{38}$ reported that there was no evidence of a link between serum ferritin and LDL oxidation parameters in either men or women in the USA (mean age 59 years) without existing myocardial infarction or history of ischaemic heart attack.

In the Transition and Health during Urbanisation of South Africans (THUSA) study conducted 10 years ago in the same population, Aderibigbe et al reported that serum ferritin concentration was significantly higher in the high WHR category than in the normal WHR category. This observation was retained in women only after adjustment was made for age, $\mathrm{BMl}$ and smoking ${ }^{39}$ This is congruent with the findings of the present study, as WHR increased significantly from the second to the fourth quartile of ferritin. The same trend was observed for WC in the present study. This suggests that central obesity contributes significantly to increased serum ferritin concentration of women in this population.

Ferritin, an acute-phase reactant, is elevated during inflammation. ${ }^{15}$ Obesity, now recognised as an inflammatory condition, ${ }^{40}$ may contribute to the elevated ferritin levels observed in this population. Hepcidin, the hormone that inhibits the release of iron from the stores and reduces absorption of iron, has been shown to be elevated in obese individuals. ${ }^{15}$ However, in the present study, obese women did not have lower iron status. Adiposity, especially centralised adipose tissue, has been reported to promote atherogenetic reactions by interacting with other CVD risk factors. ${ }^{14}$ Crist et al reported that markers of lipid damage such as oxidised low-density lipoprotein (oxLDL) and 15-isoprostane $\mathrm{F}_{2 \alpha}\left(\mathrm{PGF}_{2}\right)$, and not markers of DNA or protein damage, contribute significantly to the development of CVD. ${ }^{5}$ This supports the role of obesity in the pathogenesis of CVD. ${ }^{41} \mathrm{~A}$ high WC has been linked to increased oxLDL in both men and women. ${ }^{42}$ In this study, $31 \%$ of participants exceeded the recommended limit for WC $(<88 \mathrm{~cm})$, while $52 \%$ exceeded the reference limits for WHR $(<0.80)$. It has been hypothesised that iron overload may not be a problem in a healthy population, but that in a population with obesity and related noncommunicable diseases, iron overload may pose a major threat. ${ }^{14}$

No significant correlation was observed between serum ferritin and TfR concentration after adjusting for valid confounders. This implies that one of these iron parameters was influenced by other factors affecting iron status; for example, ferritin is affected by alcohol consumption and inflammation. ${ }^{43}$ In the present study, the mean serum ferritin concentration in drinkers $(260.42 \mu \mathrm{g} / \mathrm{l})$ was double that in non-drinkers $(122.58 \mu \mathrm{g} / \mathrm{l})$. The mean self-reported alcohol consumption in this population was $7.74 \mathrm{~g} / \mathrm{d}$, which falls within the reference range for moderate drinking. ${ }^{44}$ However, the mean selfreported alcohol intake of women drinkers was $23.30 \mathrm{~g} / \mathrm{d}$, which is way above the reference range for moderate drinking. ${ }^{4-45}$ 
The mechanism by which alcohol increases serum ferritin concentration is not fully understood. ${ }^{46}$ One of the speculated mechanisms is the leakage of intracellular ferritin by cell necrosis. ${ }^{46}$ Serum ferritin, under normal circumstances, is in equilibrium with intracellular ferritin. ${ }^{24}$ Alcohol is thought to influence this equilibrium..$^{46}$ This is supported by the observation that serum ferritin in alcoholics correlates with increased transaminase levels, which were normalised when alcohol was withdrawn. ${ }^{47}$ Alcohol may also have a direct effect on ferritin synthesis. ${ }^{46}$ Alcohol induces ferritin synthesis in rat hepatocyte primary cultures at low concentrations, which can easily be achieved in alcoholism. ${ }^{46}$

It was previously thought that iron accumulation has to be severe to cause oxidative stress, but research findings suggest that moderately excessive serum iron may contribute to formation of oxygen radicals, which can initiate oxidation of LDL. ${ }^{48}$ 0xidised LDL is a scavenger receptor and, when taken up by the macrophages can lead to the formation of foam cells, the most essential cells of the fatty streak. ${ }^{14} \mathrm{~A}$ mean serum ferritin concentration of $80 \mu \mathrm{g} / \mathrm{l}$ has been associated with significant increases in incidence of heart disease for both genders. ${ }^{28}$ In the present study, the mean serum ferritin concentration was $94.84 \mu \mathrm{g} / \mathrm{l}$. It is believed that oxidative stress itself can release the iron needed for production of reactive species. Superoxide radicals have been observed to liberate iron from ferritin, thereby inducing lipid peroxidation. It has been proposed that the role of iron in CVD involves the conversion of poorly reactive free radicals to highly reactive ones. ${ }^{29}$

\section{Conclusion}

This population-based study suggests no significant association between iron status variables and CVD risk factors except ferritin, which increased with increasing abdominal obesity, as defined by WHR and WC. This may pose a greater risk for CVD as the proportion of iron-replete individuals increases.

\section{References}

1. Halliwell B, Gutteridge JMC. Role of free radicals and catalytic metal ions in human disease: an overview. In: Packer L, Glazer AN, editors. Methods in enzymology. San Diego: Academic Press, 1990; p. 1-85.

2. Ramakrishnan U, Kuklina E, Stein AD. Iron stores and cardiovascular disease risk factors in women of reproductive age in the United States. Am J Clin Nutr. 2002;76:1256-1260.

3. Kruger HS, Kruger A, Vorster HH, et al. Urbanisation of Africans in the North West Province is associated with better micronutrient status: the Transition and Health during Urbanisation Study in South Africa. Nutr Res. 2005;25:365-375.

4. Cogswell ME, McDonnell SM, Khoury MJ, et al. Iron overload, public health, and genetics: evaluating the evidence for hemochromatosis screening. Ann Intern Med. 1998;129:971-979.

5. Crist BL, Alekal DL, Ritland LM, et al. Association of oxidative stress, iron and centralized fat mass in healthy postmenopausal women. J Womens Health (Larchmt). 2009;18:795-801.

6. Masse PG, Dosy J, Cole DEC, et al. Is serum ferritin an additional cardiovascular risk factor for all postmenopausal women? Ann Nutr Metab. 2004;48:381-389

7. Sempos CT, Looker AC, Gillum RF, et al. Serum ferritin and death from all causes and cardiovascular disease: the NHANES II Mortality Study. National Health and Nutrition Examination Study. Ann Epidemiol. 2000;10:441-448.

8. Tuomainen T, Punnonen K, Nyyssonen K, Salonen JT. Association between body iron stores and the risk of acute myocardial infarction in men. Circulation 1998;97:1461-1466.

9. Halle M, Konig D, Berg A, et al. Relationship of serum ferritin concentrations with metabolic cardiovascular risk factors in men without evidence for coronary artery disease. Atherosclerosis 1997; $128: 235-240$.

10. Morrison HI, Semeneiw RM, Mao Y, Wigle DT. Serum iron and risk of fatal acute myocardial infarction. Epidemiology 1994;5:243-246

11. Salonen JT, Nyyssonen K, Korpela H, et al. High stored iron levels are associated with excess risk of myocardial infarction in eastern Finnish men. Circulation 1992;86:803-811.

12. Steyn NP, Labadarios MB, Mauder E, et al. Secondary anthropometric data analysis of the national food consumption survey in South Africa: the double burden. Nutrition 2005;21:4-13.
13. Vorster $\mathrm{HH}$. The emergence of cardiovascular disease during urbanisation of Africans. Public Health Nutr. 2002;5:239-243.

14. Reddy MB, Clark L. Iron, oxidative stress, and disease risk. Nutr Rev. 2004;62:120-124.

15. Gillum RF. Association of serum ferritin and indices of body fat distribution and obesity in Mexican American men. The Third National Health and Nutrition Examination Survey. Int J Obes. 2001;25:639-645

16. Baynes RD. Assessment of iron status. Clin Biochem. 1996;29:209-215.

17. Cook JD, Finch CA, Smith NJ. Evaluation of the iron status of a population. Blood 1976;48:449-455.

18. Kohgo $\mathrm{Y}$, Nishisato $\mathrm{T}$, Kondo $\mathrm{H}$, et al. Circulating transferrin receptor in human serum. $\mathrm{Br} \mathrm{J}$ Haematol 1986;64:277-281.

19. Punnonen K, Irjala K, Rajamaki A. Serum transferrin receptor and its ratio to serum ferritin in the diagnosis of iron deficiency. Blood 1997;89:1052.

20. Lohman TG, Roche AF, Martorell R. Anthropometric standardisation reference manual. Champaign, Illinois: Human Kinetic Books; 1988.

21. Mbewu A. Operational plan for comprehensive HIV and AIDS care, management and treatment for South Africa. South African Government Information [homepage on the Internet]. c2007. Available from: www.info.gov.za/issues/hiv/careplan.htm

22. Friedewald WT, Levy RI, Fredrickson DS. Estimation of the concentration of low-density lipoprotein cholesterol in plasma, without use of the preparative ultracentrifuge. Clin Chem. 1972;18:499-502.

23. World Health Organisation. Obesity: preventing and managing the global epidemic. Report of WHO Consultation on Obesity, June 5-7, 1997. Geneva: WHO; 1998.

24. Centers for Disease Control and Prevention. Recommendations to prevent and control iron deficiency in the United States. MMWR Morb Wkly Rep. 1998;47(RR-3):1-29.

25. Kasdan TS. Medical nutrition therapy for anaemia. In: Malian LK, Escott-Stump S, editors. Krause's food, nutrition and diet therapy. 10 $0^{\text {th }}$ edition. London: WB Saunders; 2000:781-800.

26. De Backer G, Ambrosioni E, Borch-Johsen K, et al. Executive summary. European guidelines on cardiovascular disease prevention in clinical practice. Third Joint Task Force of European and other Societies on Cardiovascular Disease Prevention in Clinical Practice (constituted by representatives of eight societies and by invited experts). Eur Heart J. 2003;24:1601-1610.

27. Ridker PM. Clinical application of C-reactive protein for cardiovascular disease detection and prevention. Circulation 2003;107:363-369.

28. Sullivan JL. Iron and the sex difference in heart disease risk. Lancet 1981;1:1293-1294.

29. Halliwell B. Free radicals, antioxidants and human disease: curiosity, cause and consequence. Lancet 1994;344:721-724.

30. Gopane RE, Pisa PT, Vorster HH, et al. Relationships of alcohol intake with biological health outcomes in an African population in transition: the THUSA study. S Afr J Clin Nutr. 2010;23:16-21.

31. Dallogeville $\mathrm{J}$, Marecaux $\mathrm{N}$, Isorez $\mathrm{D}$, et al. Multiple coronary heart disease risk factors are associated with menopause and influence by substitutive hormonal therapy in a cohort of French women. Atherosclerosis 1995;118:123-133.

32. Van der A DL, Grobbee DE, Roest M, et al. Serum ferritin is a risk factor for stroke in postmenopausal women. Stroke 2005;36:1637-1641.

33. Milman N, Kirchhoff M. Relationship between serum ferritin and risk factors for ischaemic heart disease in 2235 Danes aged 30-60 years. J Intern Med. 1999;245:423-433.

34. Wakatsuki A, Ikenoue N, Sagar Y. Estrogen-induced small low-density liporprotein particles in postmenopausal women. Obstet Gynecol. 1998;91:234-240.

35. Tribble DL, Holl LG, Wood PD, Krauss RM. Variations in oxidative susceptibility among six low density lipoprotein subfractions of differing density and particle. Atherosclerosis 1992;93:189-199.

36. Binkoski AE, Kris-Etherton PM, Beard JL. Iron supplementation does not affect the susceptibility of LDL to oxidative modification in women with low iron status. J Nutr. 2004;134:99-103.

37. Danesh J, Appleby P. Coronary heart disease and iron status: meta-analyses of prospective studies. Circulation 1999;99:852-854

38. Irribarren C, Sempos CT, Eckfeldt JH, Folsom AR. Lack of association between ferritin level and measures of LDL oxidation: The ARIC Study. Atherosclerosis 1998;139:189-195.

39. Aderibigbe OR, Pisa PT, Ramoteme LM, et al. The relationship between indices of iron status and selected anthropometric cardiovascular disease risk factors in an African population: the THUSA study. Cardiovasc J Afr. 2011;22:1-8.

40. Yanoff LB, Serbring NG, Mchugh T, et al. Inflammation and iron deficiency in the hypoferrimia of obesity. Int J Obes. 2007;31:1412-1417.

41. Taylor A. Cataract: relationship between nutrition and oxidation. J Am Coll Nutr. 1993;12:138-146.

42. Weinbrenner T, Schroder H, Escurriol V, et al. Circulating oxidized LDL is associated with increased waist circumference independent of body mass index in men and women. Am J Clin Nutr. 2006;83:30-35.

43. Milman N, Kirchhoff M. The correlation between serum ferritin, alcohol consumption and social status in a population of 2236 Danes. Ugeskr Laeger. 1998;160:277-282.

44. Nutrition and Food Security Programme. World Health Organization (WHO) Regional Offices for Europe. Food-based dietary guidelines in the WHO European region. Copenhagen:WHO; 2003:1-38.

45. Pisa PT. Associations between biological alcohol consumption markers, reported alcohol intakes, and biological health outcomes in an African population in transition [doctoral thesis] Potchefstroom: North-West University, Centre of Excellence for Nutrition; 2008.

46. Moirand R, Lescoat G, Hubert N, et al. Alcohol induction of ferritin expression in a human hepatoblast cell line (Hep G2). Clin Exp Res. 1990;14:847-852.

47. Vilmaki M, Harkonen M, Ylimahri R. Serum ferritin and iron levels in chronic male alcoholics before and after ethanol withdrawal. Alcohol 1983;18:255-260.

48. Roest M, Van der Schouw YT, De Valk B, et al. Heterozygosity for a hereditary hemochromatosis gene is associated with cardiovascular death in women. Circulation 1999; 100:1268-1273. 